\title{
Classroom Manipulative to Engage Students in Mathematical Modeling of Disease Spread: 1+1 = Achoo!
}

\author{
H. Gaff ${ }^{1, *}$, M. Lyons ${ }^{2}$ and G. Watson ${ }^{3}$ \\ ${ }^{1}$ Department of Biological Sciences \\ ${ }^{2}$ Department of Department of Ocean, Earth and Atmospheric Sciences \\ ${ }^{3}$ Darden College of Education \\ Old Dominion University, Norfolk, Virgina 23529
}

\begin{abstract}
Infectious diseases ranging from the common cold to cholera affect our society physically, emotionally, ecologically, and economically. Yet despite their importance and impact, there remains a lack of effective teaching materials for epidemiology and disease ecology in K-12, undergraduate, and graduate curricula [2]. To address this deficit, we've developed a classroom lesson with three instructional goals: (1) Familiarize students on basic concepts of infectious disease ecology; (2) Introduce students to a classic compartmental model and its applications in epidemiology; (3) Demonstrate the application and importance of mathematical modeling as a tool in biology. The instructional strategy uses a game-based mathematical manipulative designed to engage students in the concepts of infectious disease spread. It has the potential to be modified for target audiences ranging from Kindergarten to professional schools in science, public health, policy, medical, and veterinarian programs. In addition, we've provided variations of the activity to enhance the transfer of fundamental concepts covered in the initial lesson to more complex concepts associated with vaccination and waning immunity. While 10 variations are presented here, the true number of directions in which the game might extend will only be limited by the imagination of its students [6].
\end{abstract}

Key words: Infectious disease, mathematical modeling, education, manipulatives AMS subject classification: 92B08

\footnotetext{
${ }^{*}$ Corresponding author. E-mail: hgaff@odu.edu
} 


\section{Introduction}

Infectious diseases have been a part of human existence from the beginning of time. The understanding of germ theory and the development of antibiotics greatly changed the mortality rates from infectious diseases. In 1900, 797 deaths per 100,000 were from infectious diseases, but that number dropped to 36 deaths per 100,000 in 1980 (Armstrong et al., 1999). This declining trend was reversed from 1980 through 1995 as a result of the AIDS epidemic, but declined again with the introduction of therapy for HIV [1]. Infectious diseases can have rapid and substantial impacts such as the 1918 influenza pandemic which killed more people in one year than died in World War I [3]. The economic impacts of infectious diseases are also staggering. While many people don't think much of the common cold, the overall economic impact of colds in the U.S. is nearly $\$ 40$ billion a year [4].

Laboratory-type exercises to teach about infectious diseases are uncommon because of the many ethical challenges that prohibit experiments such as introducing a live pathogen to a group of susceptible students and tracking its fate. These limitations rightly preclude the ability to study epidemiology directly, and so most classroom teaching is limited to lecture, reading, and indirect, post-event, data analyses. This makes learning about infectious diseases very fact-based and abstract, and yet, students often learn best through engaged learning such as hands-on laboratory experiments [8].

Epidemiologists will classify people according to disease status such as susceptible to the disease (S), infected and infectious (I), or recovered and immune(R). Diseases such as chicken pox and measles follow such an SIR disease course, as shown in Figure 1. Each infectious disease has its own disease course through a variety of other potential stages such as incubation periods, vaccinated, etc. Mathematical modelers often use this simple disease course classification structure to explore disease dynamics [6].

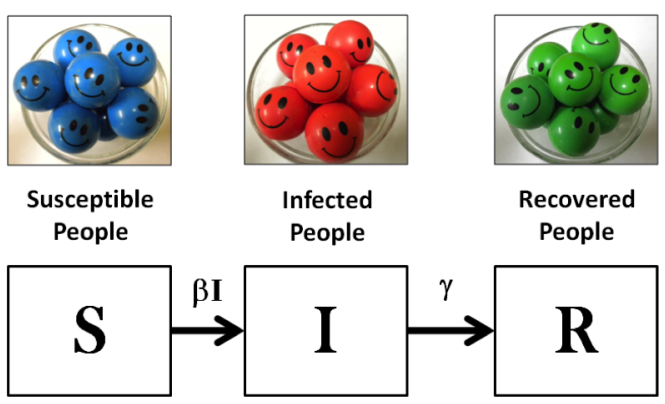

Figure 1: Simple SIR modeling tracks people in three disease states: Susceptible ( $S$; blue balls), Infected ( $I$; red balls), and Recovered ( $R$ : green balls). The rates at which people transition between susceptible and infected states depends on both the number of infected individuals $(I)$ and a transmission parameter $(\beta)$ whereas the rate at which individuals recover is constant $(\gamma)$.

The lesson presented in this paper is a way to introduce, educate, and discuss infectious diseases while demonstrating a process that can lead to a tutorial on modeling. The hands-on, game-like 
nature of the exercise allows the students to be fully engaged and even competitive. The flexibility of this exercise allows the instructor to tailor the material to any aspect of infectious disease epidemiology and to customize to specific student levels, contexts, and content.

\section{Instructional Approach}

The lesson uses the nine events of instruction [5] and integration, organization, and elaboration strategies [7] to promote learning, retention, and transfer. The lesson uses a flexible game-based guided inquiry as the primary generative learning strategy where students visualize the spread of infection under a variety of conditions starting with simple and moving to more complex examples. The lesson begins with (1) gaining attention, (2) setting expectations, and (3) stimulating recall of prior information. The lesson proceeds with the game-based exercise where the student is given (4) a context for the material, (5) guidance to organize observations and trends during the game, (6) prompts to analyze the outcomes at the end of the game, and (7) informative feedback from the teacher to confirm or refute their understanding of infectious disease spread based on the game scenario. The final steps involve (8) assessment of the student's understanding and (9) either a summary of the concepts covered to promote retention or continued play with more complex versions of the game to promote elaboration and transfer.

\section{Lesson}

Gain attention: To gain the student's attention and set the context for the lesson, ask students to raise their hands if they have had a cold. Then ask if they know where or from whom they got the cold. If time permits, ask if they have had other infectious diseases such as chicken pox. State that by the end of the lesson today, they should understand basic concepts of how infectious diseases spread from one individual to others in the hopes that they can reduce their chance of getting a cold or similar diseases in the future.

Inform students of the objective: Inform the students that they are going to play a game to see how infection is spread under a variety of conditions and that they will record what they observe. The objective at the end of the game is to be able to determine what contributes to the spread of disease and to predict the rate of spreading given different conditions.

Stimulate recall of prior information: For tracking the spread of infectious diseases in the human population, epidemiologists categorize individuals into one or more disease-related states. The states are called compartments in the classic epidemiology literature. In the simplest form, people are either susceptible (S) to the disease or infected (I) with the disease. This would represent a disease with little to no immunity such as the common cold. Diseases that confer immunity (e.g., chicken pox) require the addition of a third state for people that have recovered (R) from the disease, acquired immunity, and consequently are no longer susceptible. People transition between states (Figure 1) when a susceptible (S) individual becomes infected (I) via contact with another 
infected individual and when an infected (I) individual has recovered (R) from the disease and gains immunity to re-infection. Tracking people in these three disease states is the basis of the SIR model.

Present the game: In this mathematical manipulative, colored objects (e.g., marbles, poker chips, balls, beans, etc.) represent the three disease states (Figure 2). For example, we use blue balls for susceptible people, red balls for infected people, and green balls for recovered people. Although the specific colors are not critical, we recommend selecting colors that will correspond to matching lines on a graph in order to improve clarity and increase understanding.

If the target audience is young (K-5th), or at an introductory level (first exposure to modeling or infectious diseases), we recommend that the lesson be conducted as a class demonstration (instead of in small groups) and that 10 balls be used for population size because the conversion from the number of people in each disease state to the percentage of the population in that state is easier and the epidemic, if it occurs, will do so in a relatively shorter period of time. For older or more advanced students, the activity can be conducted in groups (as opposed to a classroom demonstration) with a set of instructions either read by the instructor or provided on an instruction sheet and a starting population of 20 to 50 objects is recommended [8]. In addition to the colored objects, each group will need an opaque container to house their population (e.g., shoe box, paper cup, large bowl, gift bag, etc.) and a means of tallying and graphing data (e.g., graph paper and colored pencils or a laptop and spreadsheet).

To start, establish the population by adding the number of susceptible "people" (e.g., 9 blue balls for beginners) and one infectious individual (e.g., 1 red ball) to the container (Figure 2). The starting conditions and underlying assumptions of the game are:

- $90 \%$ susceptible, $10 \%$ infectious, $0 \%$ immune in the initial population

- All infected individuals are infectious

- All susceptible individuals are equally likely to get infected

- One infected individual makes one susceptible individual sick upon direct contact

- Infected individuals recover at a constant rate

- All recovered individuals have immunity for life

- People who are sick still come in contact with others as usual

- There are no births, immigration, emigration, or deaths in the population

Complete the demonstration by following these game rules or by providing them to the student:

1. Randomly distribute individuals in the population by mixing the objects in the container (e.g., shaking the balls in the shoebox or stirring the marbles in the cup)

2. Blindly pull 2 objects (e.g., balls) out of the container, keeping track of what was pull each time on a table

3. If both balls are the same color, then nothing happens (put them back in the container)

4. If one ball is red (infected) and one ball is blue (susceptible), then replace the blue ball with a red ball and put both red balls back into the container 
5. Every 10 pulls (i.e., 10 contacts), replace a red ball (infected) with a green ball (recovered and immune)

6. For subsequent pulls, if the 2 balls are (blue and green) or (red and green), no disease spread occurs, so put both balls back into the container

7. For each pull (i.e., contact), graph the number of each colored object (i.e., three lines on the graph with the $\mathrm{x}$-axis as time, using the number of contacts, and the y-axis as the number of people in that disease state, as represented by the number of colored objects)

8. The game is over when there are no more red (infectious) balls in the container
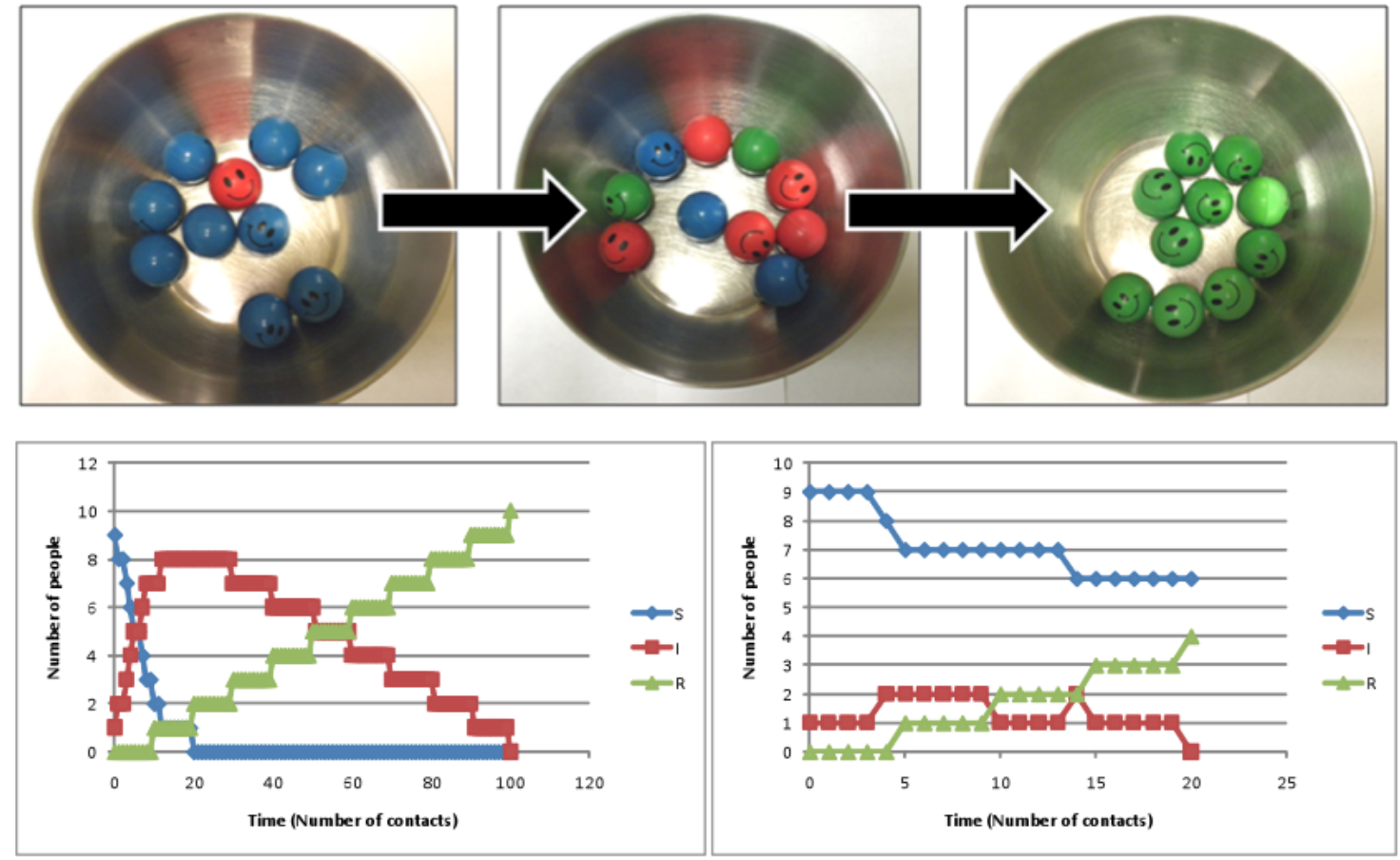

Figure 2: The activity begins with a population that is $90 \%$ susceptible (i.e., 9 blue balls) and $10 \%$ infected (i.e., 1 red ball; top left photo). If the infected individual spreads the disease to enough susceptible individuals, then an epidemic will move through the population (center photo and red line on left graph) before the population eventually recovers (right photo). A game run is over when there are no more infected individuals in the population (i.e., all green balls or a mix of green and blue balls). Occasionally, the red balls will recover before getting pulled out and consequently, no epidemic is observed (graph on right). This is realistic because one sick individual does not necessarily cause an epidemic.

Provide learning guidance: Reinforce concept formation and integration by asking what happened at the end of the game and reinforcing that under these conditions, eventually everyone 
got well. Stimulate further by asking groups to analyze how many turns or "exposures" it took before everyone was well and what trends are visible in the susceptible, infected, and recovered individuals over these runs.

The entire game should be repeated at least three times with these same conditions, either by the same group or, preferably, by three or more different groups. The results of each run and the variation among game runs should be used to generate discussion about both infectious disease ecology and SIR modeling. Specifically, the discussion should focus on variations or similarity in each of the iterations and the impact of susceptible, infectious, or immediate individuals given the assumptions in the starting conditions of model.

Elicit performance: The next step in extending this math-biology exercise is to challenge the students to evaluate the starting conditions and underlying assumptions of the game compared to what they know about specific diseases. The results that are generated while using the starting conditions are referred to as the control runs. After generating at least three control graphs, student groups conduct an experiment by proposing a single change to the starting conditions or assumptions (see potential questions/suggestions below), hypothesizing the effect of their change on the spread of the disease (i.e., the epidemic), executing the game with the change in conditions, and comparing results from their experimental runs to the control runs. The experimental runs (i.e., playing the game with any conditions other than the initial conditions) should also be completed in triplicate to observe the degree of consistency in patterns along with any variations in absolute numbers.

Game variations have been divided by difficulty. Level I questions are for young or introductory students and for class demonstrations. Level II questions are designed for more experienced participants or as a follow-up after Level I questions have been completed. Level III questions should only be attempted after students have completed the other levels or by truly advanced students (i.e., already have some training in SIR modeling and infectious disease ecology). Allow each group to select the question within their level or randomly distribute questions among groups. Each group should work together to figure out which assumption is being challenged, how to incorporate the change into the game, and what the new SIR model would look like. At the end of their experimental runs, students look at their tables and their graphs to compare the control epidemic curves (i.e., with the defined starting conditions) to the new curves both qualitatively and quantitatively in terms of how severe the peak was (highest number of infected), when the peak occurred (what pull the peak occurred at), and the duration (the width of the curve from more than $5 \%$ infected until returns to $5 \%$ infected). Groups should compare data and discuss why there was variation in results. Suggested game variations include:

\section{LEVEL I}

1. How does hygiene affect disease spread?

- New Concept: Hygiene

- Concept taught: As hygiene increases the rate of disease spread decreases

- Assumption challenged: All susceptible individuals are equally likely to get infected 
- Experimental change to game rules: When an infected (red ball) and a susceptible (blue ball) are pulled out of the container, flip a coin to determine if the susceptible person washed their hands. If "heads", then "yes" and therefore the susceptible person does not get sick (i.e., the object does not turn red, it stays blue, and is returned to the container). This can also be done with rolling dice and selecting the outcomes that result in "yes" or "no" (e.g., all even rolls = yes or the number $5=$ no).

- SIR model: new probability of infection $(\beta)$

2. How does the contagiousness of the pathogen affect the epidemic?

- New concepts: Pathogen, virulence

- Concept taught: As the contagious nature of the pathogen increases the rate of disease spread increases

- Assumption challenged: One infected individual makes one susceptible individual sick upon direct contact

- Experimental change to game rules: Instead of one infected object (red ball) making one susceptible sick, one infected object makes 2 susceptibles sick (this would represent a more contagious disease)

- SIR model: new probability of infection $(\beta)$

3. How does the starting percentage of sick people affect the epidemic?

- New concept: Inoculum size

- Concept taught: As the starting percentage of sick individuals increases the rate of disease spread increases

- Assumption challenged: $90 \%$ susceptible, $10 \%$ infectious, $0 \%$ immune in the initial population

- Experimental change to game rules: Instead of one infectious individual (i.e., one red ball) in the starting population, change the starting conditions to $20 \%$ (i.e., 2 red balls), $30 \%$, or $50 \%$ red objects to observe the effect of starting percentage on the timing, severity, and/or duration of an epidemic.

- SIR model: new numbers for initial conditions

LEVEL II

1. How does length of recovery time affect an epidemic?

- New concept: Recovery time

- Concept taught: As recovery time increases the rate of disease spread increases

- Assumption challenged: Infected individuals recover at a given rate (e.g., 10 contacts) 
- Experimental change to game rules: Vary the number of contacts required before an infected object is changed to the recovered color. This could be done systematically to increase (e.g., every 15, 20, 25 contacts) or decrease recovery time (e.g., every 2, 5, 7 contacts).

- SIR model: varying recovery rate $(\gamma)$

2. How does waning immunity affect an epidemic?

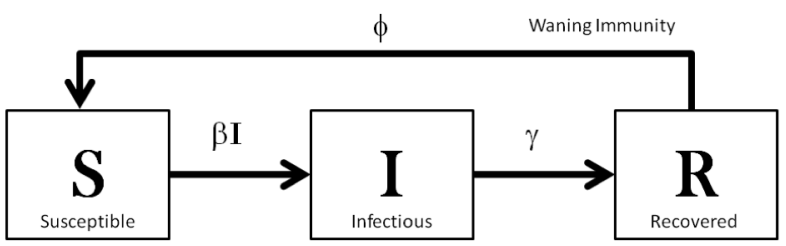

- New concept: Waning immunity

- Concept taught: As immunity decreases (i.e., waning immunity increases) the rate of disease spread increases

- Assumption challenged: All recovered individuals have immunity for life

- Experimental change to game rules: After so many contacts (i.e., pulls out of the container), a green ball is replaced with a blue ball representing the loss of immunity to the disease.

- SIR model: new arrow from $\mathrm{R}$ to $\mathrm{S}$ with new parameter

3. How does vaccination affect the epidemic?

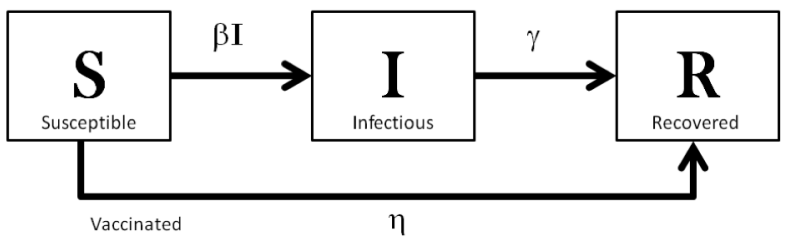

- New concept: Vaccination

- Concept taught: As vaccination increases the rate of disease spread decreases

- Assumption challenged: $90 \%$ susceptible, $10 \%$ infectious, $0 \%$ immune in the initial population 
- Experimental change to game rules: Instead of 9 blue balls in the starting population, some will start out pink (or a new color); if a pink and a blue or a pink and a red are pulled out, then nothing happens - both go back into the bowl. Observe how the presence of vaccinated people impacts the timing, severity, and/or duration of an epidemic.

- SIR model: new arrow (with new parameter) from S to R, bypassing I; therefore now both green and pink represent the " $R$ " compartment.

4. How does a latent period affect the epidemic?

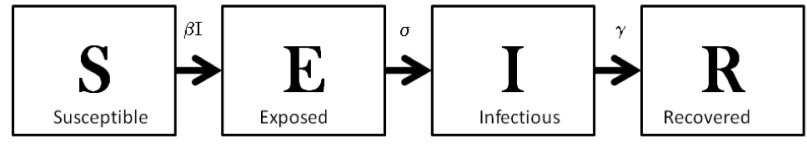

- New concept: Latent period

- Concept taught: As latent period increases, the rate of disease spread among susceptible individuals slows. As the latent period decreases the rate of disease spread increases.

- Assumption challenged: All infected individuals are infectious

- Experimental change to game rules: This would add another colored ball, such that there is a latent (orange) stage that is not infectious (red). In this case, when a red and a blue ball are pulled out, the blue is replaced with an orange. Orange balls cannot get blue balls sick (only red balls can). Orange balls stay orange for a set number of contacts before being replaced with red. The transition would then be: blue to orange to red to green, but only contact between blue and red results in further spread of the disease.

- SIR model: new category (i.e., box), now called an SEIR model

\section{LEVEL III}

1. How does the degree of waning immunity affect the epidemic?

- Concept taught: As immunity decreases (waning immunity increases) the rate of disease spread increases.

- Assumption challenged: All recovered individuals have immunity for life

- Experimental change to game rules: Vary the number of contacts (e.g., 5, 10, 15, 20) that occur before the green ball is replaced with the blue ball to observe the effect of waning immunity on the timing, severity, and/or duration of an epidemic.

- SIR model: new arrow from $\mathrm{R}$ to $\mathrm{S}$ and new rate of change

2. What percentage of the population needs to be vaccinated to prevent an epidemic? 
- Concept taught: As the percentage of vaccinated increases the chance of an epidemic decreases.

- Assumption challenged: $90 \%$ susceptible, $10 \%$ infectious, $0 \%$ immune in the initial population

- Experimental change to game rules: Start with one pink in the starting population. Then vary the number of pink balls (e.g., 2, 3, 4, 5) until an epidemic (peak) consistently does not occur.

- SIR model: new arrow from $\mathrm{S}$ to $\mathrm{R}$ and variations in the rate of change

3. How does the length of the latent period affect the epidemic?

- Concept taught: As length of the latent period increases the rate of disease spread among susceptible individuals decreases. As the latent period decreases the rate of disease spread increases.

- Assumption challenged: All infected individuals are infectious

- Experimental change to game rules: The length of time the orange stays orange before turning blue can also be evaluated to observe effects on the epidemic

- SIR model: new box and new rate of change

4. How does consistency of the recovery time affect an epidemic?

- Concept taught: The more consistent the recovery time, the more predictive the trends in the epidemic.

- Assumption challenged: Infected individuals recover at a constant rate (10 contacts)

- Experimental change to game rules: Vary the number of contacts required before an infected object is changed to the recovered color in a random fashion (recovery occurs at random time intervals)

- SIR model: new gamma

Provide feedback and assess understanding: As students run variations of the model, ask questions about the underlying assumptions in each of the exercises and provide feedback to ensure that the primary concepts are mastered, underlying assumptions are corrected, and that variations are understood relative to prior examples. This can be done using in-class discussion or through individual or group worksheets.

Enhance retention and transfer: Ask the students to collaborate and determine how each of the following might be evaluated by the game: carriers (people who spread the disease but are not obviously sick), super-spreaders (individuals that spread the disease more than others), quarantines, vectors and fomites that do not require contact between the people, fatalities, new people (immigration or births), and more or less vulnerable classes of people based on age, sex, or culture. For additional reading on SIR model variations see Hethcote [6]. 


\section{Conclusion}

We have described a simple, game-based classroom lesson that teaches many basic principles of epidemiology and mathematical modeling. Students at every level can relate to infectious diseases, but this exercise helps them understand underlying concepts associated with the entire disease process. The exercise also allows students to generate problem-based reasoning that can transfer to quantitative modeling skills without the phobias that often accompany mathematical exercises. The variations on the game allow the instructor to teach many different aspects of public health including intervention methods. The instructor can also use the exercise as a launching point for more complex mathematics using Excel or other software.

\section{Acknowledgements}

HDG thanks the editorial assistance of B. Gaff with this manuscript. HDG was supported by Grant Number K25AI067791 from the National Institute Of Allergy And Infectious Diseases. The content is solely the responsibility of the authors and does not necessarily represent the official views of the National Institute Of Allergy And Infectious Diseases or the National Institutes of Health. MML acknowledges financial support from a NOAA Interdisciplinary Research \& Training Initiative on Coastal Ecosystems \& Human Health (I-RICH) Post-Doctoral Fellowship, and NSF Ecology of Infectious Disease Grants (\#0914429/\#0914459), and the assistance of K. Lyons in developing variations of the exercise.

\section{References}

[1] G. L. Armstrong, L. A. Conn, Robert W. Pinner. Trends in Infectious Disease Mortality in the United States During the 20th Century. JAMA, 281 (1999), 61-66.

[2] C. A. Brewer, A. R. Berkowitz, P. Conrad, J. Porter, M. Waterman. Educating About Infectious Disease Ecology. In: Infectious Disease Ecology. R. S. Ostfeld, F. Keesing, V. T. Eviner (eds.), Princeton University Press, 2000, 448-466.

[3] A. Crosby. America's forgotten pandemic: the influenza of 1918 (2nd Ed). Cambridge University Press, Cambridge, UK, 2003.

[4] A. M. Fendrick, A. S. Monto, B. Nightengale, M. Sarnes. The Economic Burden of NonInfluenza-Related Viral Respiratory Tract Infection in the United States. Arch Intern Med, 163 (2003), 487-494.

[5] R. M. Gagne, K. L. Medsker. The Conditions of Learning: Training Applications. Harcourt Brace \& Co. New York, New York, 1996. 
[6] H. Hethcote. A thousand and one epidemic models. In: Frontiers in Mathematical Biology, S. Levin (ed.), Lecture Notes in Biomathematics 100, Springer, Berlin, 1994, 504-515.

[7] D. H. Jonassen. Integrating learning strategies into courseware to facilitate deeper processing. In: Instructional designs for microcomputer courseware. D. H. Jonassen (ed.), Erlbaum, Hillsdale, New Jersey, 1988, 151-182.

[8] J. R. Jungck, H. D. Gaff and A. Weisstein. Mathematical Manipulative Models: "In Defense of Beanbag Biology”. CBE-Life Sciences Education, 9 (2010), 201-211. 